\title{
Strong Complementarity and Non-locality in Categorical Quantum Mechanics
}

\author{
Bob Coecke $^{1}$ Ross Duncan ${ }^{2}$ Aleks Kissinger ${ }^{1}$ Quanlong Wang ${ }^{3}$ \\ ${ }^{1}$ University of Oxford, Department of computer science, \\ Wolfson Building, Parks Road, Oxford OX1 3QD, UK. \\ coeckelalek@cs.ox.ac.uk \\ ${ }^{2}$ Université Libre de Bruxelles, Laboratoire d'Information Quantique \\ Campus Plaine, Boulevard du Triomphe, 1050 Brussels, Belgium \\ rduncan@ulb.ac.be \\ ${ }^{3}$ Beihang University, School of Mathematics and System Sciences \\ XueYuan Road No.37, HaiDian District, Beijing, China \\ qlwang@buaa.edu.cn
}

\begin{abstract}
Categorical quantum mechanics studies quantum theory in the framework of dagger-compact closed categories.

Using this framework, we establish a tight relationship between two key quantum theoretical notions: non-locality and complementarity. In particular, we establish a direct connection between Mermin-type non-locality scenarios, which we generalise to an arbitrary number of parties, using systems of arbitrary dimension, and performing arbitrary measurements, and, a new stronger notion of complementarity which we introduce here.

Our derivation of the fact that strong complementarity is a necessary condition for a Mermin scenario provides a crisp operational interpretation for strong complementarity. We also provide a complete classification of strongly complementary observables for quantum theory, something which has not yet been achieved for ordinary complementarity.

Since our main results are expressed in the (diagrammatic) language of dagger-compact categories, they can be applied outside of quantum theory, in any setting which supports the purely algebraic notion of strongly complementary observables. We have therefore introduced a method for discussing nonlocality in a wide variety of models in addition to quantum theory.

The diagrammatic calculus substantially simplifies (and sometimes even trivialises) many of the derivations, and provides new insights. In particular, the diagrammatic computation of correlations clearly shows how local measurements interact to yield a global overall effect. In other words, we depict non-locality.
\end{abstract}

\section{INTRODUCTION}

This paper is concerned with two central notions in quantum foundations and quantum computation, non-locality and complementarity, and the study of their relationship.

Non-locality is what Einstein notoriously referred to as 'spooky action at a distance'. It was formally substantiated for the first time by Bell's theorem, and experimentally verified by testing Bell-inequalities. It states that the correlations observed when measuring spatially quantum separated systems cannot be explained by means of classical probabilities, i.e. that there is no underlying hidden variable theory. Complementarity, informally put, states that if one knows the value of one observable sharply (e.g. position), then there is complete uncertainty about the other observable (e.g. momentum).

These two concepts underpin what is arguably the most successful endeavour towards quantum information technologies: quantum cryptography. Indeed, in a quantum key distribution protocol, encoding data in either of two complementary observables will enable the parties to detect interception by an adversary [7], while non-locality allows one to verify the authenticity of the shared entangled resource by means of which key sharing is established [23].

This research applies methods of computer science and logic to investigations in quantum foundations. It is moreover strongly aligned with the current trend in the broader quantum information community: understanding quantum information processing within a larger space of hypothetical information processing theories in order to understand what is particular about quantum theory.

Until now, this area has been characterised by the study of generalised probabilistic theories [6], a space of theories which includes quantum probability theory, classical probability theory, as well as theories which are even more non-local than quantum theory. A topic of particular focus has been the search for peculiarities of quantum non-locality, within the larger space of non-local theories, e.g. [38], [5].

Of course, a space of more general theories can be conceived as abstracting away certain concrete features of quantum theory, hence encompassing a broader class of theories, and the words 'generalised' and 'abstract' can be treated as synonymous. While the generalised probabilistic theories discussed above abstract away all but convex probabilistic structure, our focus is on the compositional structure on processes, say generalised process theories.

While having composition play a leading role evidently draws form computer science practice, it also appeals to Schrödinger's conviction that what mostly characterises quantum theory is the manner in which systems compose [39].

This compositional paradigm was the main motivation for 
categorical quantum mechanics (CQM), initiated by Abramsky and Coecke in [2]. Meanwhile, CQM has helped to solve open problems in Quantum Information and Computation e.g. [14], [20], [30], and has meanwhile been adopted by leading researchers in the area of quantum foundations e.g. [9], [26].

In this paper we push CQM far beyond its previously established horizons, both on the level of comprehensiveness and in terms of its application domain. The vehicle to do so is Mermin's ingenious but elaborate non-locality proof [8], [27], [25], [34]. This argument, usually stated mostly in natural language, establishes non-locality as a contradiction of parities for quantum theory and local theories, rather than as the violation of a Bell-inequality, and involves a sophisticated interplay of several incompatible measurement scenarios, each involving measurements against varying angles, as well as hypothetical hidden variable theories, and the manipulations of the resulting probabilistic measurement data.

Our abstraction as well as our generalisations of this scenario provides important new physical insights in the nature of non-locality, and its relationship to complementarity, as we discuss in Section VIII. Hence this paper is both one in Computer Science as well as one in Quantum Foundations.

Outline. Sec. II provides an overview of CQM and presents some relevant non-standard models. We briefly recall the diagrammatic calculus for symmetric monoidal categories.

In Sec. III] we define strong complementarity, establish its relationship with ordinary complementarity, and state the first major result of this paper: the classification of strongly complementary observables in quantum theory.

In Sec. IV we diagrammatically compute the correlations of measurements against arbitrary angles on an $n$-party GHZstate for systems of arbitrary type.

In Sec. $\nabla$ we cast the usual Mermin argument within CQM, as a stepping stone to its generalisation in Sec. VI. In doing so we rely crucially on strong complementarity.

Finally, in Sec. VII we establish the necessity of strong complementarity for Mermin arguments. This also provides an operational interpretation for strong complementarity.

Earlier work. In [10], [11] Coecke and Duncan introduced the graphical ZX-calculus for the specific purpose of reasoning graphically about qubits. This calculus included the equations that we identify here as strong complementarity, but these were not identified as such. In [13] Coecke, Edwards and Spekkens relied on the CQM concept of a phase groups to identify the differences of the categories Stab and Spek with respect to non-locality, but the Mermin scenario was not formulated within CQM. In [21], [22] Edwards expanded this line work and derived some higher-dimensional generalisations of the Mermin argument.

\section{BACKGROUND I}

\section{A. Models of Physical Theories}

Symmetric monoidal categories (SMCs) provide a very general language for physical theories: a morphism $f: A \rightarrow B$ is interpreted as a process from a physical system of type $A$ to one of type $B$. Spatial and temporal extension are represented by the composition and tensor product; that is, by the sequential and parallel combination of processes. A state of system $A$ is simply a morphism $\psi: I \rightarrow A$, while an effect has type $\pi: A \rightarrow I$. Every SMC has a commutative monoid of scalars, the morphims $s: I \rightarrow I$.

Each concrete SMC is a model of this primitive theory, with different physical characteristics. For example, $($ FHilb, $\otimes)$, the category of of finite dimensional Hilbert spaces and linear maps, comprises quantum systems and pure post-selected quantum processe 1 . We write $\mathbf{F H i l b}_{D}$ to denote the category of Hilbert spaces of dimension $D^{n}$, for some fixed $D$. In particular, Qubit $:=$ FHilb $_{2}$. In fact, FHilb has redundencies: two linear maps that are equal up to a non-zero scalar multiple represent the same physical process.

This redundancy can be eliminated by using a different model. The category $C P(\mathbf{F H i l b})$ models open quantum systems and completely positive maps. It is built from FHilb using Selinger's $C P$-construction [40], described Sec. [I-E, which constructs a category of mixed processes from any given category of pure processes.

Despite its seemingly different nature, $($ FRel, $\times)$, the category of finite sets and relations with the Cartesian product as the tensor is a key example. Restricting to powers of a fixed set of size $D$ we write $\mathbf{F R e l}_{D}$.

In fact, FRel shares many features of FHilb and $C P($ FHilb). All three are compact categories [31]: each object $A$ has a dual object $A^{*}$ and morphisms $\eta_{A}: I \rightarrow A^{*} \otimes A$ and $\varepsilon_{A}: A \otimes A^{*} \rightarrow I$ such that $\left(\varepsilon_{A} \otimes 1_{A}\right) \circ\left(1_{A} \otimes \eta_{A}\right)=1_{A}$ and $\left(1_{A^{*}} \otimes \varepsilon_{A}\right) \circ\left(\eta_{A} \otimes 1_{A^{*}}\right)=1_{A^{*}}$. We refer to these elements as the compact structure on $A$. Note that they need not be unique: an object may support several compact structures.

Further, our three examples are all dagger compact [2]: there exists an identity-on-objects involutive contravariant strict monoidal endofunctor $\dagger: \mathbf{C} \rightarrow \mathbf{C}$ with $\varepsilon_{A}=\eta_{A}^{\dagger} \circ \sigma_{A^{*}, A}$ for all objects $A$, to which we refer as the dagger functor.

Dagger compactness provides the language for many quantum concepts such as Bell-states/effects, unitarity, the Born rule [2], and complete positivity [40]. E.g. unitarity means $U^{\dagger} \circ U=1_{A}$ and $U \circ U^{\dagger}=1_{B}$. An SMC with a dagger functor is referred to as $\dagger$-SMC. In any $\dagger$-SMC we can define an inner product for states $\psi$ and $\phi$ as $\psi^{\dagger} \circ \phi: I \rightarrow I$. This provides the usual inner-product (in FHilb) as well as amplitudes (in $C P(\mathbf{F H i l b})$ ). More examples of physical models can be found in Appendix A-D

\section{B. †-Compact Categories and Diagrammatic Calculus}

Monoidal categories admit a diagrammatic notation which greatly simplifies the task of reading, analysing, and computing in this framework. For the $\dagger$-compact categories of interest here this language takes a particularly simple form.

Systems (objects) are depicted by labelled wires, and processes (morphisms) are represented as boxes with wires in

\footnotetext{
1 'Pure' as in 'not mixed'; 'post-selected' means that processes which are conditional on obtaining a given measurement outcome are permitted.
} 
and out, indicating the type of the process. Composition is expressed by plugging the outputs of one box into the inputs of another, and the monoidal product is given by juxtaposition. The monoidal unit $I$ is represented as the empty diagram.

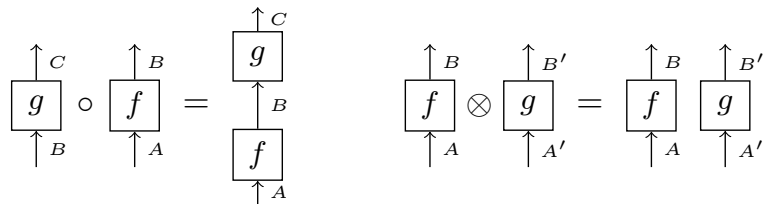

We shall draw composition from bottom-to-top, and omit wire labels where there is no ambiguity. In a symmetric monoidal category, we indicate the swap map as a wire crossing. For compact closed categories, we indicate a dual object $A^{*}$ by a wire of the opposite direction. The cap and cup maps are then depicted as half-turns.

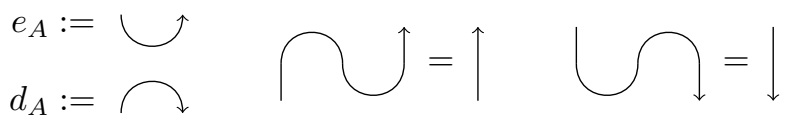

Using caps and cups, we can turn any morphism $f: A \rightarrow B$ into a morphism on the dual objects going in the opposite direction: $f^{*}: B^{*} \rightarrow A^{*}$.

$$
\stackrel{\downarrow}{f^{*}}=\sqrt{\downarrow}
$$

This is sometimes called the transpose of $f$, but this terminology can be misleading. In FHilb, $L^{*}$ is the map that takes a linear form $\langle\xi| \in B^{*}$ to $\langle\xi| L \in A^{*}$. We refer to this map simply as the upper-star of $f$. In a $\dagger$-category, the $\dagger$ functor sends $f: A \rightarrow B$ to $f^{\dagger}: B \rightarrow A$. In a †-compact category, we define the lower-star of $f$ as $f_{*}:=\left(f^{\dagger}\right)^{*}=\left(f^{*}\right)^{\dagger}$.

Since our category is symmetric, wires are allowed to cross, and boxes may slide up along wires freely without changing the denotation of the diagram. More generally, if one diagram may be deformed continuously to another, then these diagrams denote the same arrow in the category. For a more comprehensive description of graphical languages, see Selinger [41].

In the diagrams to come, we will often use horizontal separation to indicate separation in space and vertical separation to indicate separation in time. For example,

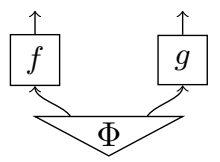

depicts the creation of two systems by the process $\Phi$, which then become spatially separated over some time and are acted upon by processes $f$ and $g$ respectively.

\section{Generalised Observables}

An observable yields classical data from a physical system [17], [16]. In quantum mechanics, an observable is represented by a self-adjoint operator. The important information encoded by a (non-degenerate) observable is its orthonormal basis of eigenstates. In FHilb, orthonormal bases (ONBs) are in 1to- 1 correspondence with $\dagger$-special commutative Frobenius algebras [18].

Definition 2.1: In a $\dagger$-SMC, a $\dagger$-special Frobenius algebra (†-SCFA) is a commutative Frobenius algebra

$$
\begin{aligned}
& \mathcal{O}_{\circ}=\left(\mu_{\circ}: X \otimes X \rightarrow X, \quad \eta_{\circ}: I \rightarrow X,\right. \\
& \left.\delta_{\circ}: X \rightarrow X \otimes X, \quad \epsilon_{\circ}: X \rightarrow I\right)
\end{aligned}
$$

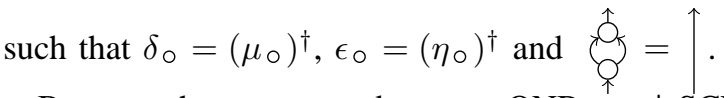

Because the correspondence to ONBs, a $\dagger$-SCFA is also called an observable structure. We will use the symbolic representation $\left(\mu_{\circ}, \eta_{\circ}, \delta_{\circ}, \epsilon_{\circ}\right)$ and the pictorial one

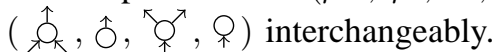

The key distinction between classical and quantum data is that classical data may be freely copied and deleted while this is impossible for quantum data, due to the no-cloning [19], [44] and no-deleting [36] theorems. This is a general fact: any compact closed category with natural diagonal maps $\Delta: X \rightarrow X \otimes X$ collapses to a preorder [1].

Proposition 2.2: Given an observable structure $\mathcal{O}_{\circ}$ on $X$, let $(\bigcirc)_{n}^{m}$ denote the ' $(n, m)$-legged spider':

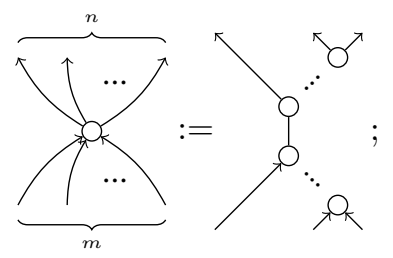

then any morphism $X^{\otimes n} \rightarrow X^{\otimes m}$ built from $\mu_{\circ}, \eta_{\circ}, \delta_{\circ}$ and $\epsilon_{\circ}$ via $\dagger-S M C$ structure which has a connected graph is equal to the $(\bigcirc)_{n}^{m}$. Hence, spiders compose as follows:

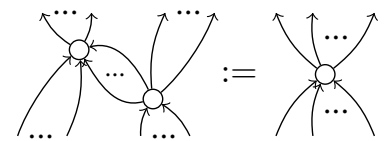

Concretely, given an ONB $\{|i\rangle\}_{i}$ then $\delta_{\circ}::|i\rangle \mapsto|i i\rangle$ defines an observable, and all observables are of this form for some ONB. The resulting intuition is that $\delta_{\circ}$ is an operation that 'copies' basis vectors, and that $\epsilon_{\circ}$ 'erases' them [16].

Perhaps surprisingly, FRel also has many distinct observables, which have been classified by Pavlovic [37]. Even on the two element set there are two [12], namely $\delta_{\circ}:\{0,1\} \rightarrow$ $\{0,1\} \times\{0,1\}:: i \mapsto(i, i)$ and $\delta_{\circ}:: 0 \mapsto\{(0,0),(1,1)\} ; 1 \mapsto$ $\{(0,1),(1,0)\}$. In fact, this pair is strongly complementary in the sense of Sec. III.

Each observable structure comes with a set of classical points, the abstract analogues to eigenvectors of an observables. A classical point is a state that is copied by its comultiplication and deleted by the counit:

$$
\underset{\substack{1 \\ i}}{\uparrow}=\underset{i}{i} \uparrow \quad \frac{i}{i}=1_{I}
$$

We will depict classical points as triangles of the same colour as their observable structure. Each observable structure 
furthermore defines a self-dual †-compact structure on its object. That is, it defines a $\dagger$-compact structure where the dual object of $X$ is $X$ itself. By (1) we have:

$$
\overbrace{0}^{\mathrm{O}} \uparrow=\overbrace{0}^{\mathrm{O}} \uparrow=\uparrow
$$

The upper-star with respect to this compact structure corresponds in FHilb to transposition in the given basis. For that reason, we call this the 0 -transpose $f^{\circledast}$. The lower star corresponds to complex conjugation in the basis of $\mathcal{O}_{\circ}$, so we call it the $\bigcirc$-conjugate $f_{\circledast}:=\left(f^{\circledast}\right)^{\dagger}$.

\section{Phase Group for an Observable Structure}

Given an observable structure $\mathcal{O}_{\circ}$ on $X$, the multiplication $\mu_{\circ}$ puts a monoid structure on the points of $X$. If we restrict to those points $\psi_{\alpha}: I \rightarrow A$ where $\mu_{\circ}\left(\psi_{\alpha} \otimes\left(\psi_{\alpha}\right)_{\circledast}\right)=\eta_{\circ}$, we obtain an Abelian group $\Phi_{\circ}$ called the phase group of $\mathcal{O}_{\circ}$ [10]. We let $\psi_{-\alpha}:=\left(\psi_{\alpha}\right)_{\circledast}$ and represent these points as circles with one output, labelled by a phase.

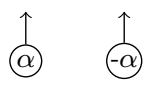

For an observable on the qubit, the phase group consists of the points that are unbiased to the eigenstates of the observable, and their multiplication is their convolution. Explicitly, given $\mu_{\circ}=|0\rangle\langle 00|+| 1\rangle\langle 11|$, we have:

$$
\mu_{\circ}\left(\left(\begin{array}{c}
1 \\
e^{i \alpha}
\end{array}\right) \otimes\left(\begin{array}{c}
1 \\
e^{i \beta}
\end{array}\right)\right)=\left(\begin{array}{c}
1 \\
e^{i(\alpha+\beta)}
\end{array}\right),
$$

so we obtain the circle group.

We can now introduce 'spiders decorated with phases':

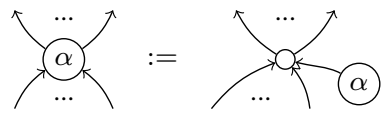

which compose as follows:

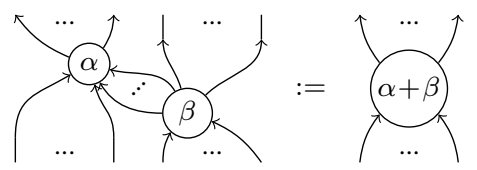

The name 'phase group' comes from fact that phased spiders with one input and one output are the abstract analogue to phase gates, familiar from quantum computing. This is supported by the following fact, proven in [11].

Proposition 2.3: If $\psi_{\alpha} \in \Phi_{\circ}$ then $\mu_{\circ} \circ\left(1_{X} \otimes \psi_{\alpha}\right): X \rightarrow X$ (i.e. a spider with one input and one output, labelled by $\alpha$ ) is unitary.

\section{E. Generalised Classical-Quantum Interaction}

In ordinary quantum theory, quantum states are represented as positive operators and operations as completely positive maps, or CPMs. These are maps that take positive operators to positive operators. A general CPM can be written in terms of a set of linear maps $\left\{B_{i}: \mathcal{H}_{1} \rightarrow \mathcal{H}_{2}\right\}$ called its Kraus maps.

$$
\Theta(\rho)=\sum_{i} B_{i} \rho B_{i}^{\dagger}
$$

Since FHilb is compact, we can regard $\rho: \mathcal{H}_{1} \rightarrow \mathcal{H}_{1}$ as an element of $\mathcal{H}_{1}^{*} \otimes \mathcal{H}_{1}$.

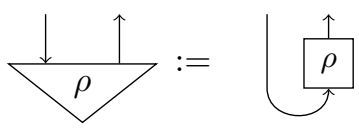

Then, we can encode the Kraus vectors of $\Theta$ in a map $B^{\prime}=\sum|i\rangle \otimes B_{i}$ and represent $\Theta$ as:

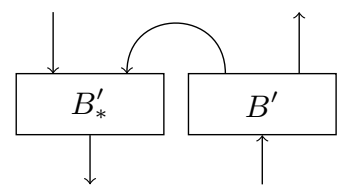

When we take the elements in Eq. (6) to be morphisms in an arbitrary $\dagger$-compact category, this gives us an abstract definition of a completely positive map. This is Selinger's representation of CPMs [40].

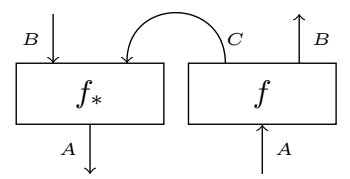

Important special cases are states where $A \cong I$, effects where $B \cong I$, and 'pure' maps, where $C \cong I$.

Returning to quantum mechanics, we can see how a quantum measurement would look in this language. A (projective) quantum measurement $M_{\circ}$ is a CPM that sends trace 1 positive operators (in this case quantum states) to trace 1 positive operators that are diagonal in some ONB (encoding a probability distribution of outcomes). Suppose we wish to measure with respect to $\mathcal{O}_{\circ}$, whose classical points form an ONB $\left\{\left|x_{i}\right\rangle\right\}$. The probability of getting the $i$-th measurement outcome is computed using the Born rule.

$$
\operatorname{Prob}(i, \rho)=\operatorname{Tr}\left(\left|x_{i}\right\rangle\left\langle x_{i}\right| \rho\right)=\left\langle x_{i}|\rho| x_{i}\right\rangle
$$

We can encode this map from states to distributions as:

$$
M_{\circ}(\rho)=\sum_{i}\left(\left\langle x_{i}|\rho| x_{i}\right\rangle\right)\left|x_{i}\right\rangle\left\langle x_{i}\right|
$$

Or, rather than encoding the distribution in a diagonal matrix, we could simply use a vector:

$$
m_{\circ}(\rho)=\sum_{i}\left(\left\langle x_{i}|\rho| x_{i}\right\rangle\right)\left|x_{i}\right\rangle
$$

Expanding this graphically, we have:

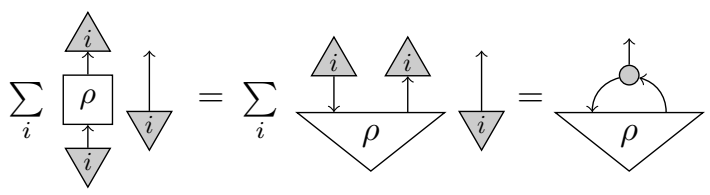

We are now ready to make definitions for abstract measurements and abstract probability distributions, which we shall call Born vector.

Definition 2.4: For an observable structure $\mathcal{O}_{\circ}$, a measurement is defined as the following map:

$$
m_{\circ}:=\jmath_{\nwarrow}
$$


Any point $\mid \Gamma): I \rightarrow X$ of the following form is called a Born vector, with respect to $\mathcal{O}_{0}$ :

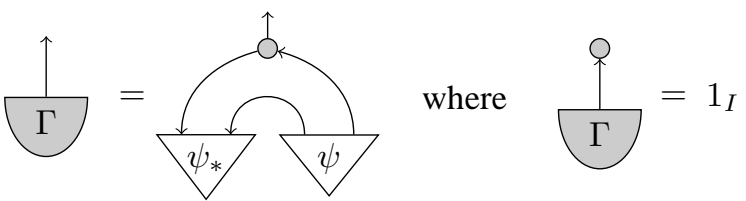

We can naturally extend the definition above to points of the form $\mid \Gamma): I \rightarrow X \otimes \ldots \otimes X$ by requiring that they be Born vectors with respect to the product Frobenius algebra $\mathcal{O}_{\circ} \otimes \ldots \otimes \mathcal{O}_{0}$.

The adjoint of the measurement map $m_{\circ}^{\dagger}$ is a preparation operation. In FHilb, it takes a Born vector $\mid \Gamma$ ) with respect to $\mathcal{O}_{\circ}$ and produces a probabilistic mixture of the (pure) outcome states of $\mathcal{O}_{0}$ with probabilities given by $\left.\mid \Gamma\right)$.

This leads to a simple classical vs. quantum diagrammatic paradigm that applies to arbitrary observables in any †-SMC [16]: classical systems are encoded as a single wire and quantum systems as a double wire. The same applies to operations, and $m_{\circ}$ and $m_{\circ}^{\dagger}$ allow passage between these types.

Note that the classical data will 'remember' to which observable it relates, cf. the encoding $\sum_{i} p_{i}\left|x_{i}\right\rangle$. This is physically meaningful since, for example, when one measures position the resulting value will carry specification of the length unit in which it is expressed. If one wishes to avoid interconversion of this 'classical data with memory', one could fix one observable, and unitarily transform the quantum data before measuring. Indeed, if

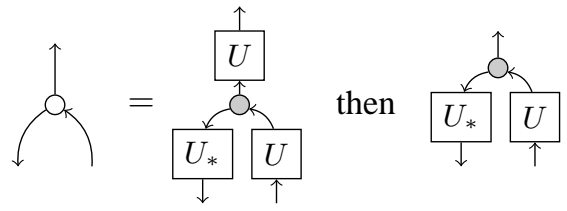

measures the $\mathcal{O}_{\circ}$-observable but produces $\mathcal{O}_{\circ}$-data. In FHilb, all observable structures are unitarily isomorphic, so any projective measurement can be obtained in this way. A particularly relevant example is when these unitaries are phases with respect the another observable structure $\mathcal{O}_{\circ}$.

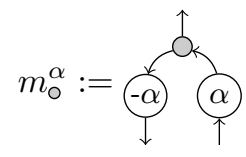

When $\mathcal{O}_{\circ}$ is induced by the Pauli spin- $Z$ observable and $\mathcal{O}_{\circ}$ by the Pauli spin- $X$ observable, then $m_{\circ}=m_{\circ}^{0}$ is an $X$ measurement and $m_{\circ}^{\pi / 2}$ is a $Y$ measurement. Note however, that both produce Born vectors of outcome probabilities with respect to the $\bigcirc$ basis. This will be useful in the sections to come.

\section{STRONG COMPLEMENTARITY}

Definition 3.1: A pair $\left(\mathcal{O}_{0}, \mathcal{O}_{0}\right)$ of observables on the same object $X$ is complementary iff:

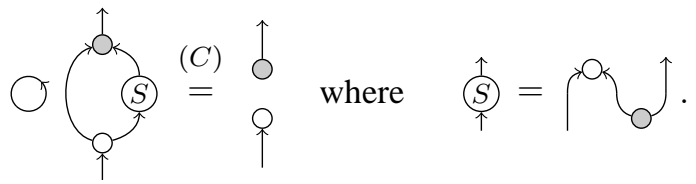

If at least one of the two observables has 'enough classical points', this equation holds if and only if the classical points of one observable are 'unbiased' (in sense of [10]) for the other observable. Every observable in FHilb has enough classical points, hence we reclaim the usual notion of quantum complementarity, and extend it to a more general setting.

Definition 3.2: A pair $\left(\mathcal{O}_{0}, \mathcal{O}_{0}\right)$ of observables on the same object $X$ is coherent iff:

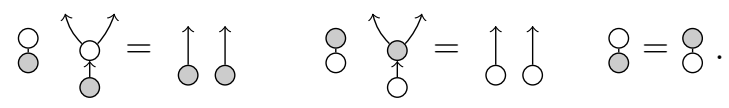

In other words, $\epsilon_{\circ}$ is proportional to a classical point for $\mathcal{O}_{\circ}$, and vice versa.

$$
O \underset{i}{i}=\uparrow \quad O \underset{i}{i}=\uparrow
$$

We will assume that the scalar $Q$ is always cancelable.

Proposition 3.3: In FHilb if $O_{\circ}$ and $O_{\circ}$ are self-adjoint operators correspoding to complementary observables, one can always choose a pair of coherent observable structures $\left(\mathcal{O}_{\circ}, \mathcal{O}_{\circ}\right)$ whose classical points correspond to the eigenbases of $O_{\circ}$ and $O_{\circ}$.

Proof: (sketch) The eigenbasis of a non-degenerate selfadjoint operator is only determined up to global phases. For a pair of mutually unbiased bases, it is always possible to choose these phases such that coherence is satisfied.

For this reason we will from now on assume that pairs of complementary observables are always coherent.

Definition 3.4: A pair $\left(\mathcal{O}_{\circ}, \mathcal{O}_{\circ}\right)$ of observables on the same object $X$ is strongly complementary iff they are coherent and:

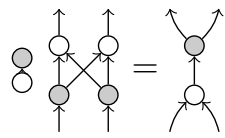

Viewing one observable as monoid and the other as comonoid, the properties of coherence and strong complementarity state that a strongly complementary pair $\left(\mathcal{O}_{\circ}, \mathcal{O}_{\circ}\right)$ form a scaled bialgebra; that is, the defining equations of a bialgebra [43] hold upto a scalar multiple.

The following results about the antipode for a strongly complementary pair were shown in [32].

Lemma 3.5: Under the assumption that classical points are self-conjugate in their own colour, and provide 'enough points', the antipode $S$ is self-adjoint, and is a Frobenius algebra endomorphism in both colours.

In fact we can go further.

Theorem 3.6: Strong complementarity $\Rightarrow$ complementarity. Proof: 


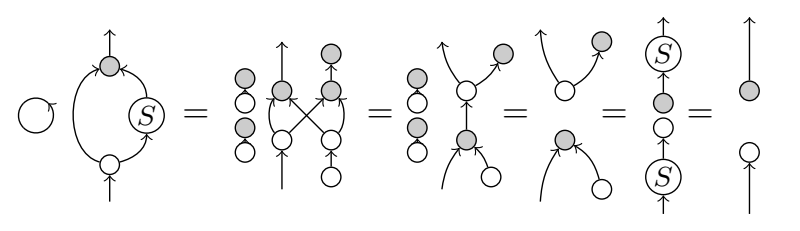

As a consequence, strongly complementary observables always form a scaled Hopf algebra. Note that Theorem 3.6 relies on the fact that both the monoid and the comonoid form a Frobenius algebra; it is certainly not the case that every scaled bialgebra is a Hopf algebra.

The converse to Theorem 3.6 does not hold: it is possible to find coherent complementary observables in FHilb which are not strongly complementary. See [11] for a counterexample.

\section{A. Strong Complementarity and Phase Groups}

For complementary observables, classical points of one observable are always included in the phase group of the other observable, up to a normalizing scalar. Strong complementarity strengthens this property to inclusion as a subgroup. Let $\mathcal{K}_{\circ}$ be the set of classical points of $\mathcal{O}_{0}$ multiplied by the scalar factor 8 .

Theorem 3.7: Let $\left(\mathcal{O}_{\circ}, \mathcal{O}_{\circ}\right)$ be strongly complementary observables and let $\mathcal{O}_{0}$ have finitely many classical points. Then $\mathcal{K}_{\circ}$ forms a subgroup of the phase group $\Phi_{\circ}$ of $\mathcal{O}_{\circ}$. The converse also holds when $\mathcal{O}_{\circ}$ has 'enough classical points'.

Proof: By strong complementarity it straightforwardly follows that, up to a scalar, $\mu_{\circ}$ applied to two classical points of $\mathcal{O}_{\circ}$ yields again a classical point of $\mathcal{O}_{\circ}$ :

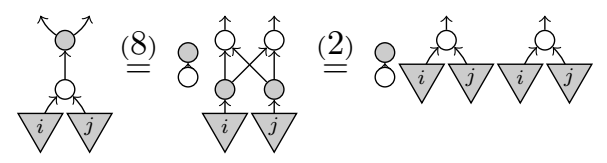

The unit of $\Phi_{\circ}$ is, up to a scalar, also a classical point of $\mathcal{O}_{\circ}$ by coherence. Hence, $\mathcal{K}_{\circ}$ is a submonoid of $\Phi_{\circ}$ and any finite submonoid is a subgroup. The converse follows from:

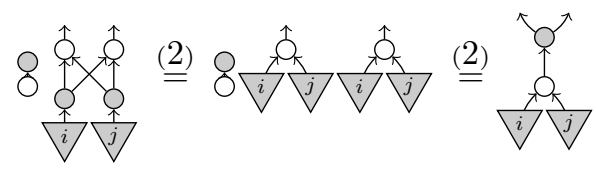

together with the 'enough classical points' assumption.

Recall that the exponent of a group $G$ is the maximum order of any element of that group: $\exp (G)=\max \{|g|: g \in G\}$.

Corollary 3.8: For any pair of strongly complementary observables, let $k=\exp \left(\mathcal{K}_{\circ}\right)$. Then, assuming $\mathcal{O}_{\circ}$ has 'enough classical points':

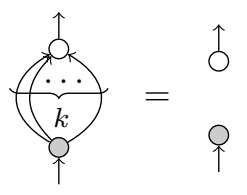

Proof: In a finite abelian group, the order of any element divides $\exp \left(\mathcal{K}_{\circ}\right)$. The result then follows by:

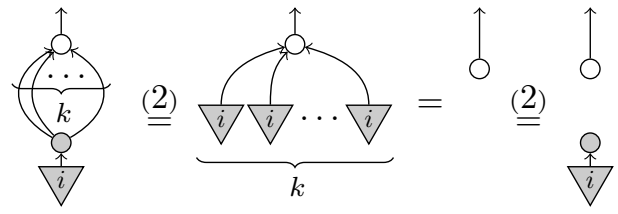

together with the 'enough classical points' assumption.

Proposition 3.9: For a pair of strongly complementary observables (i) is a $\mathcal{O}_{0}$-homomorphism for all $(i) \in \mathcal{K}_{0}$. Conversely, this property defines strong complementarity provided $\delta_{\circ}$ has 'enough classical points'.

Proof: Similar to the proof of Thm. 3.7 .

\section{B. Classification of Strong Complementarity in FHilb}

Corollary 3.10: Every pair of strongly complementary observables in FHilb is of the following form:

$$
\left\{\begin{array} { l } 
{ \delta _ { \circ } : : | g \rangle \mapsto | g \rangle \otimes | g \rangle } \\
{ \epsilon _ { \circ } : : | g \rangle \mapsto 1 }
\end{array} \quad \left\{\begin{array}{l}
\delta_{\circ}^{\dagger}::|g\rangle \otimes|h\rangle \mapsto \frac{1}{\sqrt{D}}|g+h\rangle \\
\epsilon_{0}^{\dagger}:: 1 \mapsto \sqrt{D}|0\rangle
\end{array}\right.\right.
$$

where $(G=\{g, h, \ldots\},+, 0)$ is a finite Abelian group. Conversely, each such pair is always strongly complementary.

Proof: By Theorem 3.7 it follows that the classical points of one observable (here $\mathcal{O}_{0}$ ) form a group for the multiplication of the other observable (here $\delta_{0}^{\dagger}$ ), and in FHilb this characterises strong complementarity.

One of the longest-standing open problems in quantum information is the characterisation of the number of pairwise complementary observables in a Hilbert space of dimension $D$. In all known cases this is $D+1$, and the smallest unknown case is $D=6$. We now show that in the case of strong complementarity this number is always 2 for $D \geq 2$.

Theorem 3.11: In a Hilbert space with $D \geq 2$ the largest set of pairwise strongly complementary observables has size at most 2.

Proof: Assume that both $\left(\mathcal{O}_{\circ}, \mathcal{O}_{\circ}\right)$ and $\left(\mathcal{O}_{\circ}, \mathcal{O}_{\bullet}\right)$ are strongly complementary pairs. By coherence $\hat{\delta}$ and $\hat{\boldsymbol{\theta}}$ must be proportional to classical points of $\mathcal{O}_{\circ}$. If $\left(\mathcal{O}_{\circ}, \mathcal{O}_{\bullet}\right)$ were to be strongly complementary observables, it is easily shown that $\boldsymbol{\delta} \neq 0$ so $\hat{\delta}$ and $\hat{\boldsymbol{\phi}}$ are proportional to the same classical point. Hence, up to a non-zero scalar:

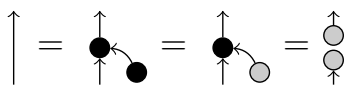

i.e. the identity has rank 1 , which fails for $D \geq 2$. By Corollary 3.10 a strongly complementary pair exists for any $D \geq 2$.

\section{Diagrammatic COMPutation OF GHZ Measurement Outcome Distributions}

In order to present a graphical Mermin/GHZ style arguement, we show how to compute measurement outcomes for an $n$-party GHZ state graphically. This computation relies crucially on the following corollary, which follows from strong complementarity via a standard theorem about bialgebras. 
Corollary 4.1: The following equation holds for any connected bipartite graph with directions as shown.

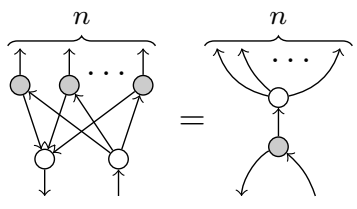

The proof is given in Appendix $\mathrm{A}-\mathrm{A}$

We compute the classical probability distributions $\left(=\mathcal{O}_{0}\right.$ data) for $n$ measurements against arbitrary phases $\alpha_{i} \in \Phi_{\circ}$ on $n$ systems of any type in a generalised $G H Z_{\circ}^{n}$-state:

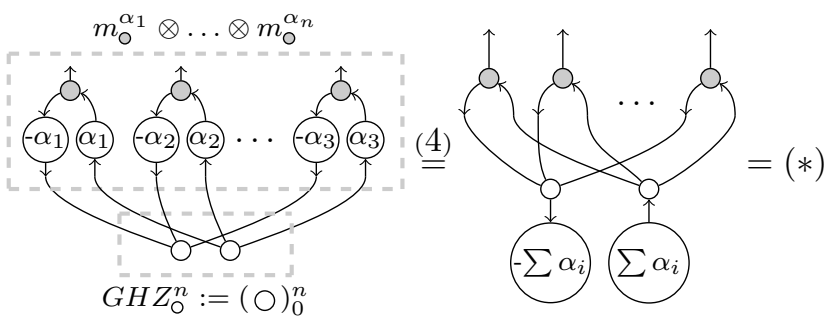

Applying Corollary 4.1, we note that this is a probability distribution followed by a $\bigcirc$-copy.

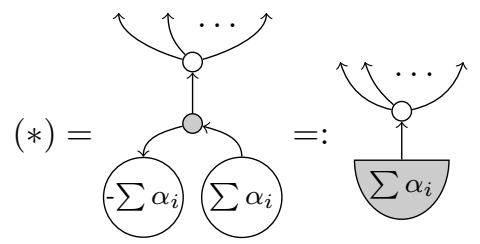

The following is an immediate consequence.

Theorem 4.2: When measuring each system of a $G H Z_{A}^{n}$ state against an arbitrary angle then the resulting classical probability distribution over outcomes is symmetric.

Theorem 4.3: The classical probability distributions for $m_{\circ}^{\alpha_{1}} \otimes \ldots \otimes m_{\circ}^{\alpha_{n}}$-measurements on a GHZ $Z_{A}^{n}$-state is:

- uncorrelated if $\left.\mid \sum \alpha_{i}\right)$ is a classical point for $\mathcal{O}_{\circ}$ and,

- parity-correlated if $\left.\mid \sum \alpha_{i}\right)$ is a classical point $i$ for $\mathcal{O}_{\circ}$ (i.e. contains precisely those outcomes $i_{1} \otimes \ldots \otimes i_{n}$ such that the sum of group elements $\sum i_{k}$ is equal to $i$ ).

Example 4.4: We can compute the outcome distributions for $X X X, X Y Y, Y X Y$, and $Y Y X$ measurements on three qubits in a GHZ-state using the technique described above. First, outcome distribution $\mid A)$ for $X X X$ :

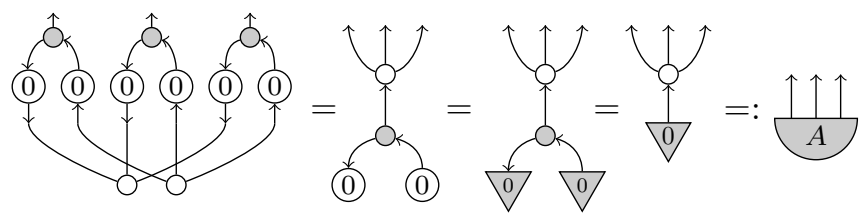

Next, we compute outcome distribution $\left.\mid B_{1}\right)$ for $X Y Y$ :

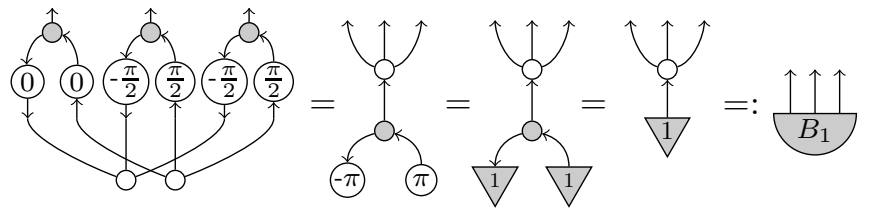

Clearly, the other two cases, $Y X Y$ and $Y Y X$, will give the same result. We therefore set $\left.\left.\left.\mid B_{1}\right)=\mid B_{2}\right)=\mid B_{3}\right)$.

\section{MERmin's Non-LOCALITy ARgument IN CQM}

For a particular $n$-party state $|\Psi\rangle$ in some theory, a local hidden variable $(L H V)$ model for that state consists of:

- a family of hidden states $|\lambda\rangle$, each of which assigns for any measurement on each subsystem a definite outcome,

- and, a probability distribution on these hidden states,

which simulates the probabilities of that theory. We say that a theory is local if each state admits a LHV model.

The Mermin argument is an important thought experiment which rules out the possibility that the predictions of quantum mechanics could be explained by LHV models [34]. Unlike Bell's argument, which merely shows that there exist quantum mechanical states whose outcome probabilities are inconsistent with locality assumptions, the Mermin argument demonstrates how there exists a quantum state whose outcome possibilities are inconsistent with locality.

Consider three systems and four possible (compound) measurement settings, consisting of the control $X X X$, and three variations $X Y Y, Y X Y$, and $Y Y X$. Let:

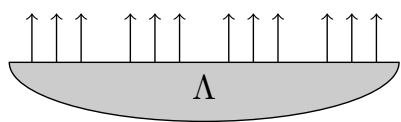

be a Born vector for $\mathcal{O}_{\circ}$ which represents the probability distribution on possible outcomes for each of these settings. This Born vector is a probability distribution over 'hidden states' each of which specifies the outcomes of all three systems for each of the four measurement settings, for example:

$$
|\lambda\rangle=|\underbrace{+--}_{X X X} \underbrace{+++}_{X Y Y} \underbrace{--+}_{Y X Y} \underbrace{-+-}_{Y Y X}\rangle
$$

yields outcomes $(+1,-1,-1)$ when $X X X$ is measured, outcomes $(+1,+1,+1)$ when $X Y Y$ is measured.

Assume now that this Born vector arises from an underlying LHV model (L). A hidden state of the LHV model stores one measurement outcome for each setting on each system:

$$
\left|\lambda^{\prime}\right\rangle=|\overbrace{\underbrace{+}_{\text {system } 1} \overbrace{-}^{X}}^{Y} \overbrace{\underbrace{X}_{\text {system } 2} \overbrace{+}^{Y}}^{\overbrace{-}^{X} \overbrace{+}^{Y}}\rangle
$$

We can represent a probability distribution over such hidden states as a Born vector $\left.\mid \Lambda^{\prime}\right)$, from which we generate $\mid \Lambda$ ) via copy maps, which put a hidden state $\left|\lambda^{\prime}\right\rangle$ into the form $|\lambda\rangle$ :

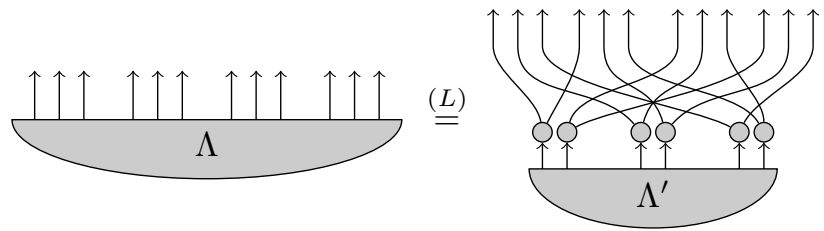

For example, the leftmost wire coming from $\left.\mid \Lambda^{\prime}\right)$ represents the outcome when the first qubit of each hidden state is measured in $X$. This outcome is copied and sent to the first qubit for setting $X X X$ and the first qubit for setting $X Y Y$.

We now investigate whether measurement of $X X X, X Y Y$, $Y X Y$ and $Y Y X$ on the qubits in a GHZ state is consistent (C) with such a LHV model. Although it is impossible to 
perform all four measurement setups simultaneously on the three qubits in the GHZ state, it should at least be true that any possible hidden state in $\mid \Lambda)$ must not be ruled out by the combined experimental data.

Since we are assuming that $\left.\left.\left.|A| \otimes \mid B_{1}\right) \otimes \mid B_{2}\right) \otimes \mid B_{3}\right)$ is the probability distribution yielded by sampling $\mid \Lambda$ ) independently for each measurement setup, it is a course-graining of $\mid \Lambda$ ) itself. In other words, the support of $\mid \Lambda)$ must be contained in the support of $\left.\left.\left.|A| \otimes \mid B_{1}\right) \otimes \mid B_{2}\right) \otimes \mid B_{3}\right)$ In particular, if we can apply some function on hidden states in $\left.|A| \otimes \mid B_{1}\right) \otimes$ $\left.\left.\mid B_{2}\right) \otimes \mid B_{3}\right)$ that yields a definite outcome, that function must yield the same outcome in $\mid \Lambda$ ). Consider the function:

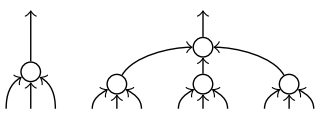

This function computes the parity (i.e. the $\mathbb{Z}_{2}$-sum) of outcomes for each of the four experiments. It then furthermore computes the overall parity of outcomes for the 3 variation experiments. We can straightforwardly show this yields a constant outcome on $\left.\left.\left.|A| \otimes \mid B_{1}\right) \otimes \mid B_{2}\right) \otimes \mid B_{3}\right)$ :

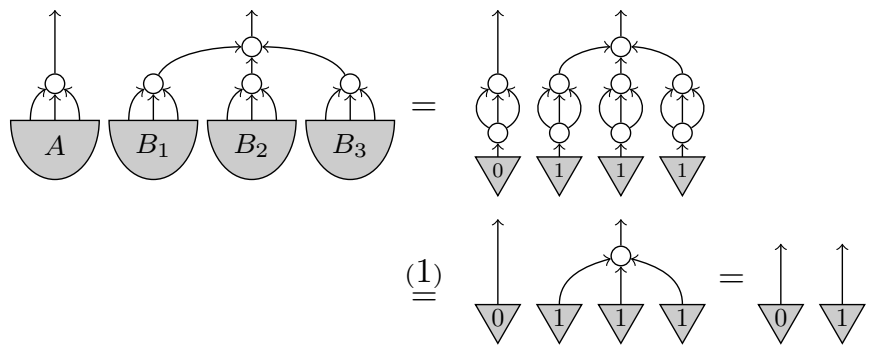

The last equation follows because by Theorem 3.7 the classical points for $\mathcal{O}_{\circ}$ form a subgroup of the phase group $\Phi_{\circ}$. Since there are two classical points for $\mathcal{O}_{\circ}$, this group must be $\mathbb{Z}_{2}$.

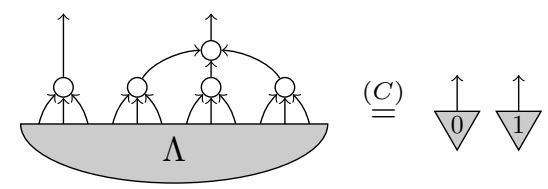

We can then use equations $(C)$ and $(L)$ to derive a contradiction. First, substitute $(L)$ into the LHS of $(C)$ :
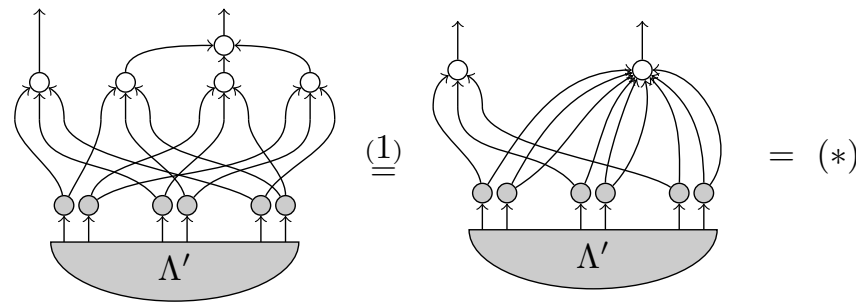

Since the group associated with $\bigcirc$ is $\mathbb{Z}_{2}$, Cor. 3.8 applies for $k=2$. Thus, we can delete pairs of parallel edges connecting dots of different colours. Then,

\footnotetext{
${ }^{2}$ Note that this is a statement about possibilities rather than probabilities.
}

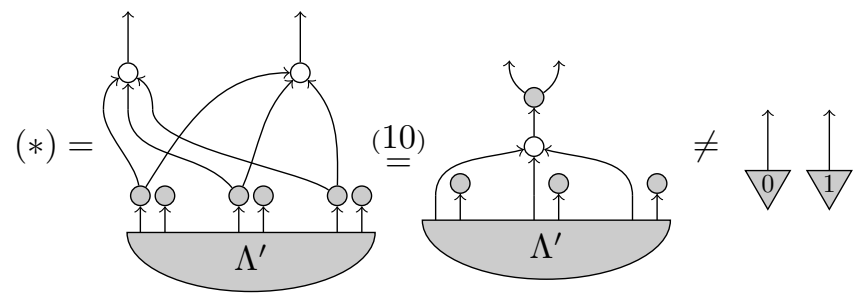

The final inequation follows from the distinctness of classical points and the cocommutativity of " $O$ '. Note that the symmetry from the LHS arises from the fact that after the application of Cor. 3.8 only the $\mathrm{X}$ measurements in the variations contribute to the output of the function.

\section{Generalised Non-Locality Arguments}

To summarise, the conflict exposed in the previous section arises from the fact that experimental data, here from quantum theory, excludes a property imposed on the hidden states by locality. That property was symmetry. We now generalise this.

First we consider more general lists of measurement settings, represented again by a Born vector for $\mathcal{O}_{0}$ generated from a LHV model:

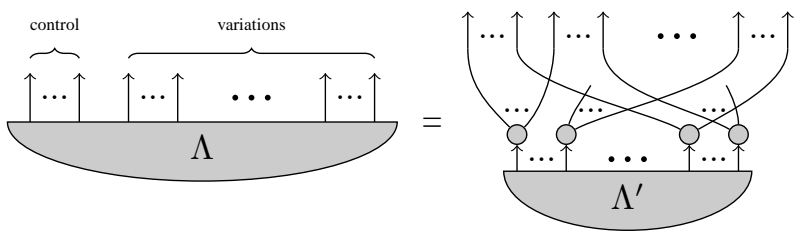

where we require, that via application of Cor. 3.8, we again obtain a symmetric expression:

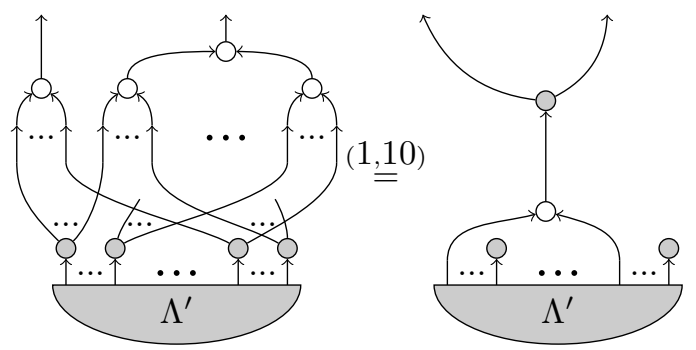

This requires that for the variations, the multiplicity of occurrence of a particular measurement on the same system has to be a multiple of $\exp \left(\mathcal{K}_{\circ}\right)$.

Contradicting this symmetry requires an inequation between classical points:

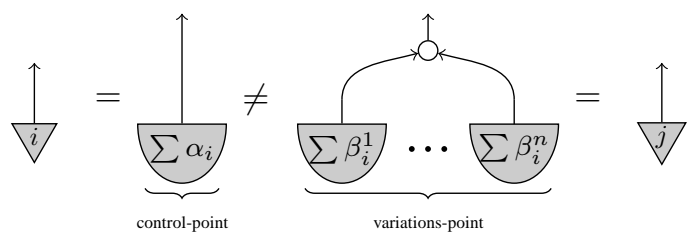

Theorem 6.1: The above scenario provides a generalised Mermin non-locality argument whenever:

1) the multiplicity of occurrence of measurements on the same system in the variations is a multiple of $\exp \left(\mathcal{K}_{0}\right)$,

2) and, the control-point and the variations-point are distinct classical points. 
This theorem yields a wide variety of generalised Mermintype non-locality arguments, and also characterises situations where such an argument fails to hold.

\section{NeCESSity of StRong COMPLEMENTARity}

In this section, we make an argument that the assumptions of the Mermin argument necessitate the use of strongly complementary observables. Consider a fixed 3-party GHZ-state $(\mathrm{O})_{0}^{3}$. We will attempt to construct a Mermin argument by measuring all three systems with respect to some observables $\mathrm{O}_{1}, \mathrm{O}_{2}, \mathrm{O}_{3}$. We make three assumptions about $O_{i}$.

1) Phase-related: All three $O_{i}$ are within a $\mathcal{O}_{0}$-phase of some fixed observable structure $\mathcal{O}_{0}$.

2) Coherence: $\mathcal{O}_{\circ}$ is coherent with respect to $\mathcal{O}_{\circ}$.

3) Sharpness: After performing two of the three measurements on the GHZ-state, the remaining system is in an eigenstate of the third measurement.

The first condition is satisfied by the usual Mermin argument, and can be seen as requiring that measurements differ in the 'maximally non-local' manner, since $\mathcal{O}_{\circ}$-phases can pass freely through the GHZ state. In FHilb, when $\mathcal{O}_{\circ}$ is already complementary, we can choose it to be coherent by 3.3 The third assumption was highlighted by Mermin in [34] as an important aspect of the experimental setup.

The map projects from the space of all quantum mixed states to the the space of classical mixtures of eigenstates of $\mathcal{O}_{0}$. To assert sharpness, we require that, once two of the three systems are measured, the third is invariant under this map:

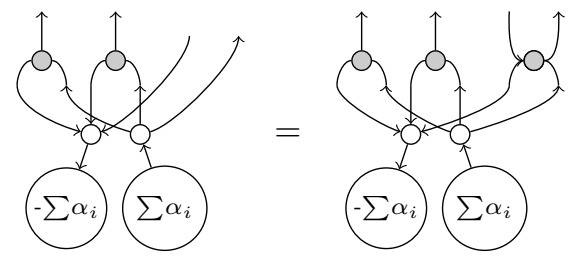

Plugging the unit of $\mathcal{O}_{\circ}$ in the 2nd system both for LHS and RHS, and using coherence we obtain:

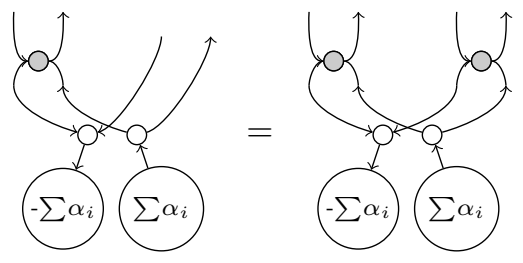

and by exploiting symmetry we have:

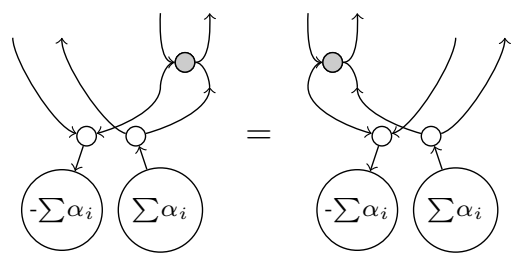

Hence we obtain:

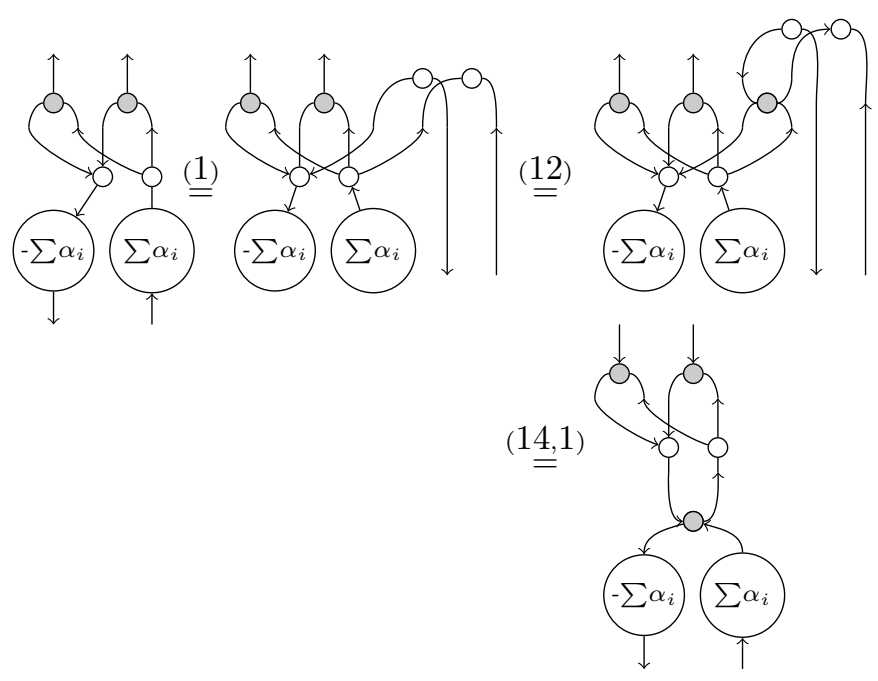

Since $\delta_{\circ}^{\dagger} \circ\left(1_{X} \otimes \sum_{i} \alpha_{i}\right)$ is unitary it cancels.

Proposition 7.1: For a pair $\left(\mathcal{O}_{\circ}, \mathcal{O}_{\circ}\right)$ of coherent observables on the same object the following equation implies (8):

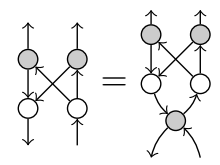

Proof: See Appendix A-B

Thus, for any coherent pair of observables, the sharpness of the third outcome necessitates strong complementarity.

\section{CONCLUSION AND OUTLOOK}

We cast Mermin's non-locality argument within CQM. This enabled us to substantially generalise this result to multiple parties, arbitrary angles and systems of arbitrary dimension.

The tools of CQM made most computations very easy as compared to the methods of standard quantum theory. We only rely on two simple rules: (i) contraction of labeled nodes (cf. (4)); (ii) commutation of the multiplications and comultiplications associated with distinctly colored nodes (cf. (10)).

In the graphical language, the manner in which the phases associated to the measurements interact through the GHZ-state effectively depicts non-locality.

The concepts required for reproducing Mermin-style nonlocality arguments as well as the derivations crucially relied on the newly introduced notion of strong complementarity. We provided an operational interpretation for strong complementarity and classified strongly complementary observables in the case of quantum theory.

Our analysis also provides new insights in Mermin's argument. For example, while we crucially rely on 'strong' complementarity between the observable that characterises the GHZ state and the one for which the classical points form a subgroup of the corresponding phase group, complementarity between the $\mathrm{X}$ and $\mathrm{Y}$ obsevables is not at all essential when passing to the general case.

The results in this paper moreover open the door for a research program that concentrates on studying general process 
theories, in order to better understand what is so peculiar about the correlations encountered in quantum theory. CQM indeed provides an ideal arena for relating key concepts of quantum theory, and investigating in which manner they survive when passing to more general process theories.

The results in this paper may also have more direct applications to quantum computing. In particular, quantum secret sharing (QSS) [29] is a protocol which very closely resembles Mermin's non-locality argument. It relies on $X$ or $Y$ measurements on a qubit in a GHZ state, and the resulting correlations. Clearly the results in this paper would enable one to substantially generalise this schemes in a similar manner that we generalised Mermin's non-locality argument. Also, [28] contains initial investigations for a CQM-treatment of a wide range of quantum informatic protocols, which could be given a similar treatment and may lead to more generalisations of quantum communication schemes.

\section{APPENDIX A \\ ADDITIONAL BACKGROUND}

\section{A. Some categorical background}

We assume that the reader is familiar with basics of category theory, and it comfortable with the notion of symmetric monoidal category (SMC). Suitable introductions to the subject are [3], [15]. We assume throughout that all our monoidal categories are strict, i.e. the morphisms $\alpha, \rho$, and $\lambda$ are identities.

Definition A.1: A dagger category is a category $\mathcal{C}$ equipped with a dagger functor $\dagger: \mathcal{C}^{\text {op }} \rightarrow \mathcal{C}$ which is (i) involutive, and (ii) acts as the identity on objects.

Definition A.2: In any dagger category, a morphism $f$ : $A \rightarrow B$ is called unitary if $f^{\dagger} \circ f=1_{A}$ and $f \circ f^{\dagger}=1_{B}$.

Definition A.3: Let $\psi, \phi: I \rightarrow A$ be points of some object. We define the inner product by $\langle\psi \mid \phi\rangle:=\psi^{\dagger} \circ \phi$.

This definition coincides with usual one in the category of finite dimensional Hilbert spaces.

Definition A.4: A dagger symmetric monoidal category ( $\dagger-$ $\mathrm{SMC})$ is a symmetric monoidal category $(\mathcal{C}, \otimes, I, \alpha, \rho, \lambda, \sigma)$ which is equipped with a strict monoidal dagger functor, such that the symmetry isomorphism $\sigma$ is unitary ${ }^{3}$.

A primary method of this paper is the use of internal algebraic structures defined inside monoidal categories. We now introduce these, one piece at a time.

Definition A.5: A monoid in $\mathcal{C}$ is a triple $(X, \mu: X \otimes X \rightarrow$ $X, \eta: I \rightarrow X)$ such that the following diagrams commute:

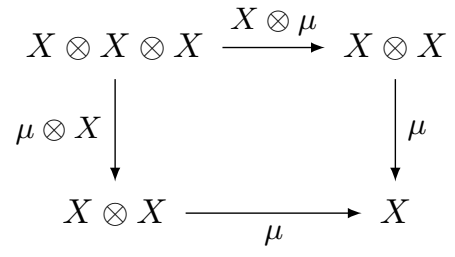

\footnotetext{
${ }^{3}$ In a non-strict setting we would insist that the other structure morphisms likewise be unitary.
}
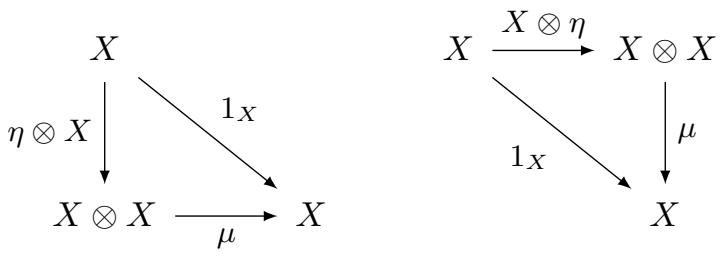

If $\mathcal{C}$ is symmetric then the monoid is commutative if it further satisfies:

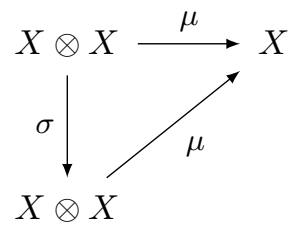

In diagrammatic notation we write $\mu=\underset{1}{n}, \eta=\hat{\jmath}$; the equations can now be expressed as
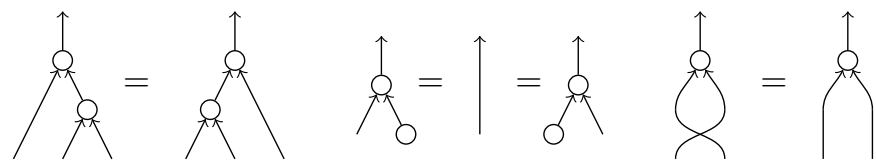

The dual to a monoid is a comonoid.

Definition A.6: A comonoid in a monoidal category $\mathcal{C}$ consists of a triple $(X, \delta, \epsilon)$ which is a monoid in $\mathcal{C}^{\text {op }}$; i.e. $\delta: X \rightarrow X \otimes X$ and $\epsilon: X \rightarrow I$ satisfy the equations of Definition $A .5$ but in reverse, viz:

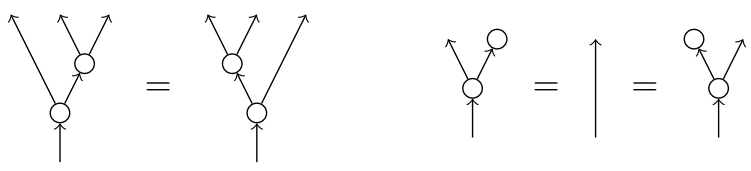

A comonoid is cocommutative if it satisfies:<smiles>CCOCCOC</smiles>

The basic example of a comonoid is in the category of finite dimensional Hilbert spaces, where for any basis $\left\{x_{i}\right\}_{i}$ of a space $X$ we obtain a comonoid by defining 'copying' and 'erasing' maps:

$$
\delta: x_{i} \mapsto x_{i} \otimes x_{i} \quad \epsilon: x_{i} \mapsto 1
$$

as the comultiplication and counit.

Notice that in a $\dagger$-SMC, if $(X, \delta, \epsilon)$ is a comonoid then automatically $\left(X, \delta^{\dagger}, \epsilon^{\dagger}\right)$ is a monoid, and vice versa.

Definition A.7: Given a comonoid $(\delta, \epsilon)$ on $X$, a comonoid homomorphism is a map $f: X \rightarrow X$ such that

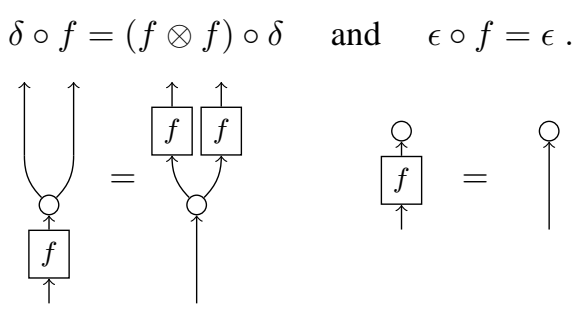

A monoid homomorphism is defined dually. 
The structures of greatest interest for this paper are algebras containing both monoids and comonoids.

Definition A.8: A commutative Frobenius algebra is a 5tuple $(X, \delta, \epsilon, \mu, \eta)$ where

1) $(X, \delta, \epsilon)$ is a cocommutative comonoid;

2) $(X, \mu, \eta)$ is a commutative monoid; and,

3) $\delta$ and $\mu$ satisfy the following equations:

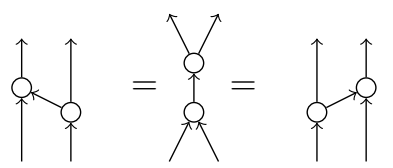

A Frobenius algebra is called special if it additionally satisfies:<smiles>COCCOC</smiles>

Let $\delta_{n}: X \rightarrow X^{\otimes n}$ be defined by the recursion

$$
\delta_{0}:=\epsilon \quad \delta_{n+1}:=\left(\delta_{n} \otimes 1_{A}\right) \circ \delta
$$

and define $\mu_{m}$ analogously. Now we have the following important theorem:

Theorem A.9: Given a SCFA $(X, \delta, \epsilon, \mu, \eta)$ let $f: X^{\otimes m} \rightarrow$ $X^{\otimes n}$ be a map constructed from $\delta, \epsilon, \mu$ and $\eta$ whose graphical form is connected. Then $f=\delta_{n} \circ \mu_{m}$.

This theorem is the basis of the 'spider' notation introduced in Proposition 2.2 in the main text.

Definition A.10: In a $\dagger-\mathrm{SMC}$, a dagger special commutative Frobenius algebra ( $\dagger$-SCFA) is a triple $(X, \delta, \epsilon)$ such that $\left(X, \delta, \epsilon, \delta^{\dagger}, \epsilon^{\dagger}\right)$ is a special commutative Frobenius algebra.

It has been shown [18] that in FHilb every †-SCFA arises from a comonoid defined by copying an orthonormal basis as described above. Since orthonormal bases define non-degenerate quantum observables, $\dagger$-SCFAs are also called observable structures.

The other structures of interest, bialgebras and Hopf algebras also arise via the interaction of a monoid and a comonoid.

Definition A.11: A bialgebra in a symmetric monoidal category is a 5-tuple $(X, \delta, \epsilon, \mu, \eta)$, where

1) $(X, \delta, \epsilon)$ is a comonoid,

2) $(X, \mu, \eta)$ is a monoid, and

3) the following equations are satisfied:

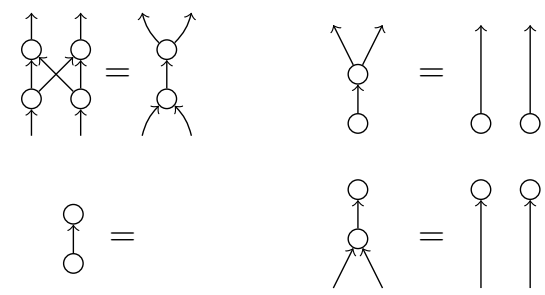

where the empty diagram stands for $1_{I}$.

In this paper, all the bialgebras we encounter will have the additional property of being Hopf algebras.

Definition A.12: A Hopf algebra in a symmetric monoidal category is a 6-tuple $(X, \delta, \epsilon, \mu, \eta, s)$ where $(X, \delta, \epsilon, \mu, \eta)$ is a bialgebra, $s: X \rightarrow X$ is map called the antipode, which altogether satisfy the equations:

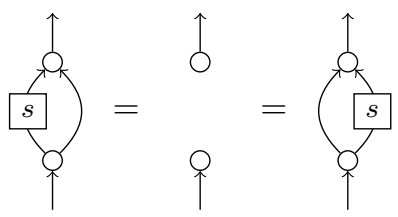

Theorem A.13: Suppose $(X, \delta, \epsilon, \mu, \eta)$ is a bialgebra and let $f: A^{\otimes m} \rightarrow A^{\otimes n}$ be a map constructed from $\delta, \epsilon, \mu$, and $\eta$. Then $f$ factorises $c \circ s \circ m$ where $c$ is constructed from the comonoid structure, $s$ is a permutation, and $m$ is constructed from the monoid structure.

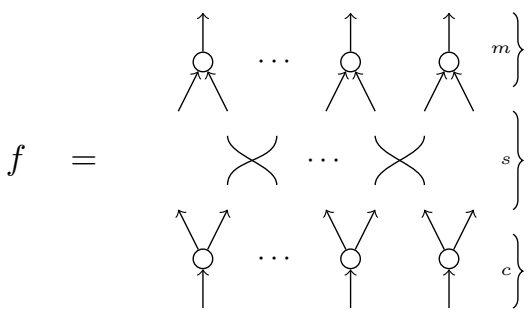

Further, this factorisation is essentially unique.

We shall not present the full proof here; for that see [33] and refernces therein. Instead we treat only the case of interest, as needed for Corollary 4.1. Suppose that $f$ has the form $\delta_{n} \circ \mu_{n^{\prime}}$; we use induction on both $n$ and $n^{\prime}$. The base case $n=0, n^{\prime}=$ 0 , i.e. $f=\epsilon \circ \eta$ is trivial. If $n=0$ and $n^{\prime}>0$ then $f$ has the form $\epsilon \circ \mu_{n^{\prime}}$ by induction on $n^{\prime}$ is equal to $n^{\prime}$ copies of $\epsilon$.

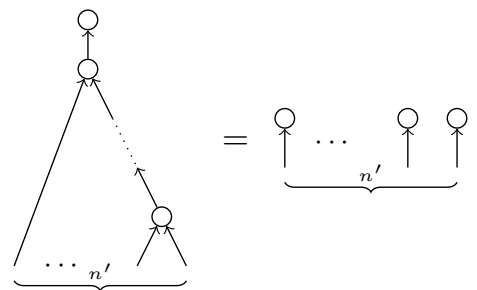

The case $n>0, n^{\prime}=0$ is exactly dual. Now suppose $n, n^{\prime}>$ 0 ; we have the situation pictured below.

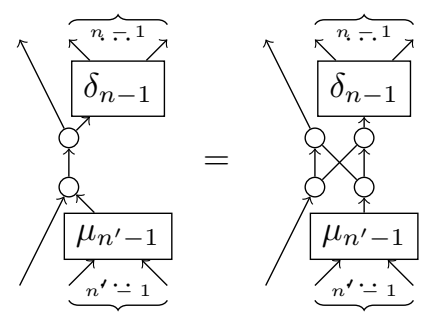

Notice that we have $\delta_{n-1} \circ \mu$ in the top left; by induction we obtain the form show below:

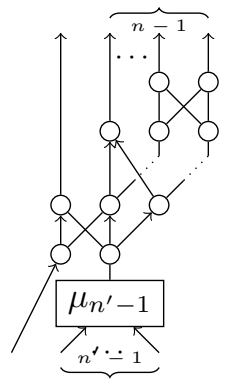


Similarly, we have $\delta \circ \mu_{n-1}$ on the bottom right and by the same reasoning we can reduce $f$ to the required form. Notice that in both diagrams there is a path from every input to every output. This preservation of paths is the defining characteristic of the normal form for bialgebras.

We can now apply the theorem to prove Corollary 4.1

Proof of Corollary 4.1. For the theorem on bialgebras to apply, all of the edges need to be directed upward. For a strongly complementary observable, the edge direction between two different colours can be reversed by applying the dualiser. Then, we use the fact that $S$ is a Frobenius algebra endomorphism to move it down.

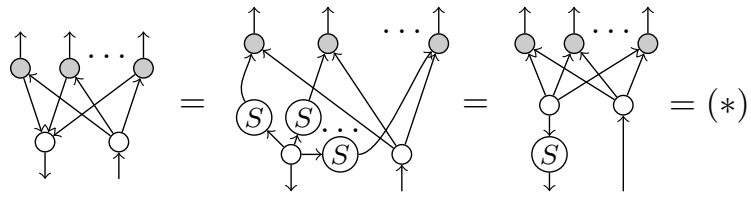

We apply Theorem A.13 and the spider theorem to complete the proof.

(*)

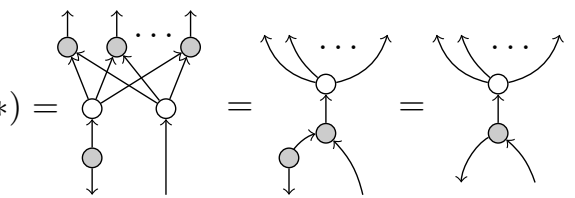

\section{B. Proof of Proposition 7.1}

We now turn to the proof of Proposition 7.1, which stated that the following equation implies strong complementarity for a pair of coherent observables $\left(\mathcal{O}_{\circ}, \mathcal{O}_{\circ}\right)$.

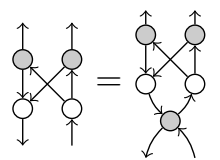

Lemma A.14: Equation (16) implies the following, for any pair of coherent observables:

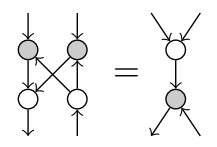

Proof:

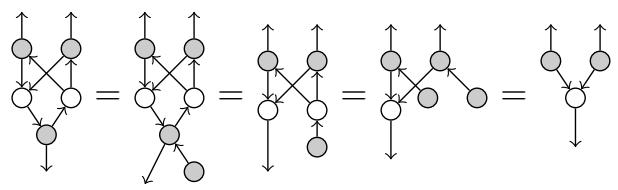

This implies:

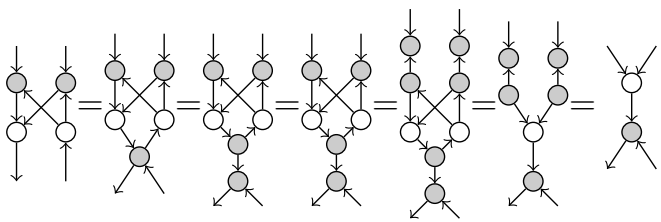

Lemma A.15: Equation (16) implies the following, for any pair of coherent observables:

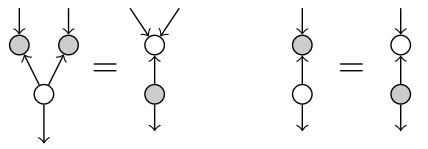

Proof:

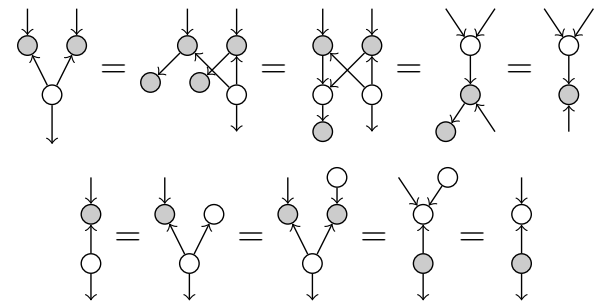

Proof of Proposition 7.1 .

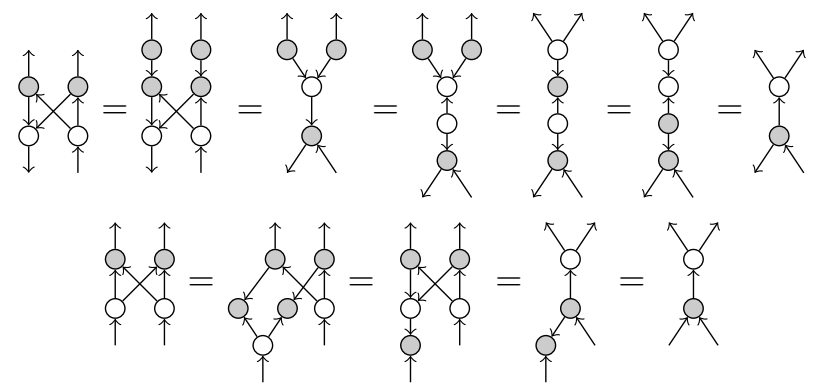

Thus any coherent pair of observable structures satisfying Equation (16) is a strongly complementary pair.

\section{Some QM background}

In this section we present the rudiments of quantum mechanics, as required by the main text.

The state space of a quantum system is a complex Hilbert space $X$; all the systems we consider here will have finite dimensional state spaces, as is typical in quantum computation. The possible states of the system are unit vectors in $X$. We use the Dirac bra-ket notation, writing vectors as $|\psi\rangle$ and their duals as $\langle\psi|$. If $X$ is $n$ dimensional, we pick a standard, or computational, basis and write its elements $|0\rangle, \ldots|n\rangle$. Therefore the standard basis for the qubit - the ubiquitous 2dimensional system used in quantum computation - is simply $|0\rangle,|1\rangle$. Another basis often seen is the $X$-basis:

$$
|+\rangle:=\frac{1}{\sqrt{2}}(|0\rangle+|1\rangle) \quad|-\rangle:=\frac{1}{\sqrt{2}}(|0\rangle-|1\rangle) .
$$

States with the vectors which differ only by a complex unit factor are physically indistiguishable so the state space is quotiented by the equivalence relation

$$
|\psi\rangle \sim|\phi\rangle \quad \Leftrightarrow \quad \exists \alpha:|\psi\rangle=e^{i \alpha}|\phi\rangle .
$$

Such a scalar factor is called a global phase.

If a quantum system is a composite of two subsystems, having state spaces $X$ and $Y$ respectively, then the state space of the compound system is the tensor product $X \otimes Y$ of the two sub-state spaces. When writing vectors of compound systems 
we may suppress the tensor product, i.e. $|00\rangle=|0\rangle \otimes|0\rangle$. Notice that $\operatorname{dim}(X \otimes Y)=\operatorname{dim}(X) \operatorname{dim}(Y)$. This has profound consequences in the form of quantum entanglement. Mathematically, entanglement is the fact that there exist states in $X \otimes Y$ which do not decompose into a pair of states from each subsystem. For example, if we consider two qubits, there are no states $|\psi\rangle,|\phi\rangle$ such that

$$
|00\rangle+|11\rangle=|\psi\rangle \otimes|\phi\rangle .
$$

This simple mathematical fact encodes many of the counterintuitive properties of quantum systems, including the nonlocal correlations which are the main concern of this paper, and the apparent speed-up seen in certain quantum algorithms.

The other crucial ingredient of the quantum formalism is measurement. Unlike classical systems, the quantum state may not be observed directly, and may only be accessed via its observable properties. Further, not every measurement has a definite result on every state: usually the result of a measurement will be probabilistic. Formally, an observable on system $X$ is a self-adjoint operator $M: X \rightarrow X$; the possible observed values of the observation are the eigenvalues of $M$. We will assume throughout that all observables have non-degerate spectra; that is we have

$$
M=\sum_{i=1}^{n} \lambda_{i}\left|e_{i}\right\rangle\left\langle e_{i}\right|
$$

where all the $\lambda_{i}$ are distinct. If we perform the measurement $M$ upon some state $|\psi\rangle$ the probability of observing $\lambda_{i}$ is given the absolute square of the inner product:

$$
p\left(\lambda_{i}\right)=\left|\left\langle e_{i} \mid \psi\right\rangle\right|^{2} .
$$

Note that since we only consider 'nice' observables, we will often identify the observable with the basis of its eigenvectors, and speak of, for example, "measuring in the computational basis".

The second shocking property of quantum measurement is the phenomenon known as the 'collapse of the wave packet'. Less mystically put, measurement changes the state of the system. Precisely, after the measurement $M$ has been performed, and the value $\lambda_{i}$ observed, the state of the system is now $\left|e_{i}\right\rangle$, the eigenvector corresponding to the outcome. Therefore, repeated measurement of the same observable will produce consistent results. For present purposes the actual observed values of the measurement are without importance: the only thing that matters is the label $i$ indicating which outcome occurred.

As a consequence of the state changing effect of measurement, two observables are well defined at the same time only if they have the same eigenvectors; that is, if their respective operators commute. Such observables are called compatible. In this work we are interested in observable that are as incompatible as possible: we call these complementary observables. Consider the Pauli $X$ and $Z$ spin observables:

$$
\sigma_{X}=\left(\begin{array}{cc}
0 & 1 \\
1 & 0
\end{array}\right) \quad \sigma_{Z}=\left(\begin{array}{cc}
1 & 0 \\
0 & -1
\end{array}\right) .
$$

Their respective eigenbases are $|+\rangle,|-\rangle$ and $|0\rangle,|1\rangle$. We have the inner products

$$
\begin{aligned}
& |\langle+\mid 0\rangle|^{2}=|\langle-\mid 0\rangle|^{2}=\frac{1}{2} \\
& |\langle+\mid 1\rangle|^{2}=|\langle-\mid 1\rangle|^{2}=\frac{1}{2}
\end{aligned}
$$

Hence when one observable is well defined, the outcomes of the other are equiprobable. Notice that if our system is in an eigenstate of $Z$, say, and we then measure $X$, the next measurement of $Z$ will be completely random. Effectively we have erased the previous value of of one observable by measuring the it with a complimentary one.

The combination of entanglement and quantum measurements gives rise to non-local correlation, which are the main subject of this paper. As a simple example, consider the 2qubit entangled state known as the Bell state,

$$
\left|\Phi_{+}\right\rangle=\frac{1}{\sqrt{2}}(|00\rangle+|11\rangle .
$$

Suppose that the two qubits of this state are in the hands of two separate parties, Aleks and Bob. If Aleks measures his qubit in the $Z$ basis, he will observe $|0\rangle$ or $|1\rangle$ with equal probability; suppose that it was $|0\rangle$. The effect of Aleks's measurement is to act on the joint system with the operator $|0\rangle\langle 0| \otimes 1$ :

$$
\begin{gathered}
(|0\rangle\langle 0| \otimes 1)(|00\rangle+|11\rangle)=(|0\rangle\langle 0| \otimes 1)|00\rangle+(\langle 0| \otimes 1)|11\rangle \\
=(|0\rangle\langle 0|| 0\rangle \otimes|0\rangle)+(|0\rangle\langle 0|| 1\rangle \otimes|1\rangle)=|00\rangle .
\end{gathered}
$$

(We neglect normalisation here.) Hence, if Bob subsequently measures his qubit he is guaranteed to observe the $|0\rangle$ outcome. Obviously if Aleks had observed $|1\rangle$ then Bob would too; the two parties measurements will be perfectly correlated.

Quantum systems which are not disturbed by measurement evolve according to unitary evolution. That is, there is some unitary operator $U: X \rightarrow X$ such that $\left|\psi\left(t_{1}\right)\right\rangle=U\left|\psi t_{0}\right\rangle$. In quantum computation these operators are usually taken to be composed of discrete quantum logic gates and it is assumed that state remains constant except when acted upon by some desired unitary gate. Composing such logic gates in sequence and parallel gives rise to the quantum circuit model. We refer the reader to [35] for details.

\section{More Physical Models}

While the main text takes FHilb as its primary model, there are a variety of other categories which support some or all of the axiomatic structures described.

For example, another $\dagger$-compact category is the category $n$ Cob, which has closed $(n-1)$-dimensional manifolds as objects, and diffeomorphism classes of $n$-dimensional manifolds connecting the objects as morphisms [4].

More generally, from a given category we can construct a new model as a subcategory. This is a surprisingly rich source of models with different properties. While all the categories discussed so far have some quantum flavour this is not necessary: modesls of classical, reversible, and stochastic processes also arise as subcategories of those already mentioned. 
For example, the subcategory $($ FSet,$\times)$ of $($ FRel,$\times)$ defined by restricting the morphisms to functions-can be viewed as a model of classical rather than quantum physics. Note that FSet is not dagger compact. Restricting the morphisms to permutations produces Perm, the category of reversible classical processes. The category of stochastic classical processes, Stoch, is also a sub-theory, this time of $C P($ FHilb), and so is FRel. In fact, as demonstrated by Coecke, Paquette and Pavlovic in [16], an analogue to the hierarchy

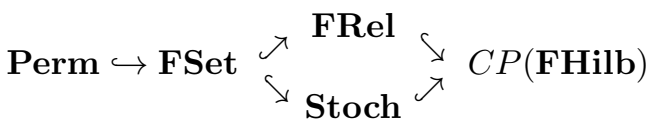

can be obtained for any $\dagger$-SMC C. It is therefore possible to extract several species of 'classical' processes from the 'quantum' ones, and to formulate notions like measurement, classical data processing and classical control of quantum systems at the abstract level of $\dagger$-SMCs, as shown in Sec. II-E

Two more quantum-like examples obtained as subcategories are the categories Stab [13] and Spek [12], which are important models in quantum computation and quantum foundations respectively.

Definition A.16: Let Stab be the subcategory of Qubit closed under $\dagger$-SMC structure and generated by 'observable' $\left(\delta: \mathbb{C}^{2} \rightarrow \mathbb{C}^{2} \otimes \mathbb{C}^{2}::|i\rangle \mapsto|i i\rangle, \epsilon: \mathbb{C}^{2} \rightarrow \mathbb{C}::|i\rangle \mapsto\right.$ 1) and Clifford group unitaries - that is those unitaries which preserve the eigenstates of the Pauli spin observables - of type $\mathbb{C}^{2} \rightarrow \mathbb{C}^{2}$.

The category Stab represents the so-called stabilizer restriction of quantum theory [24], which, roughly speaking is quantum theory were we only consider six states of a qubit, namely the $\mathrm{X}, \mathrm{Y}$ and $\mathrm{Z}$ eigenstates.

Definition A.17: The category Spek is the subcategory of $\mathbf{F R e l}_{4}$ closed under $\dagger$-SMC structure and generated by 'observable' $(\delta: 4 \rightarrow 4 \times 4:: 1 \sim\{(1,1),(2,2)\} ; 1 \sim$ $\{(1,2),(2,1)\} ; 3 \sim\{(3,4),(4,3)\} ; 4 \sim\{(3,3),(3,3)\}, \epsilon$ : $4 \rightarrow 1::\{1,3\} \sim 1)$ and the symmetric group $S(4)$.

Spek is a categorical presentation of the toy theory introduced by Spekkens [42] which tries to mimic Stab despite being local, and indeed has many features that are usually interpreted as purely quantum. While the framework of generalised probabilistic theories failed to capture Spek in any significant way, both Stab and Spek can be easily accommodated within the process theory framework.

Both categories are generated by one observable (see Sec. [II-C) and a copy of $S(4)$ of unitary operations on the smallest non-trivial system. This in itself already establishes that these theories bear a very close relationship, and moreover allowed Coecke, Edwards and Spekkens to pinpoint there essential difference [13] in terms of their phase groups, which we define below in Sec. III-D.

In the case of Stab and Spek the phase group captures their essential difference, respectively being $\mathbb{Z}_{4}$ and $\mathbb{Z}_{2} \times \mathbb{Z}_{2}$ [13]. This distinction captures all the essential differences of the two theories, in particular with respect to non-locality.

\section{REFERENCES}

[1] S. Abramsky (2009) No-Cloning In Categorical Quantum Mechanicsarxiv arXiv:0910.2401 1 .

[2] S. Abramsky and B. Coecke (2004) A categorical semantics of quantum protocols. In: Proceedings of 19th IEEE conference on Logic in computer science, pages 415-425. IEEE Press. arXiv:quant-ph/0402130 Revised version (2009): Categorical quantum mechanics. In: Handbook of Quantum Logic and Quantum Structures, K. Engesser, D. M. Gabbay and D. Lehmann (eds), pages 261-323. Elsevier. arXiv:0808.1023

[3] S. Abramsky and N. Tzevelekos (2011) Introduction to Categories and Categorical Logic. In: New Structures for Physics, B. Coecke (Ed.), pages 3-94. Springer Lecture Notes in Physics vol. 813.

[4] J. C. Baez (2006) Quantum quandaries: a category-theoretic perspective. In: The Structural Foundations of Quantum Gravity, D. Rickles, S. French and J. T. Saatsi (Eds), pages 240-266. Oxford University Press. arXiv:quant-ph/0404040

[5] H. Barnum, J. Barrett, L. O. Clark, M. Leifer, R. W. Spekkens, N. Stepanik, A. Wilce and R. Wilke (2010) Entropy and information causality in general probabilistic theories. New Journal of Physics 12, 033024. arXiv:0909.5075

[6] J. Barrett (2007) Information processing in generalized probabilistic theories. Physical Review A 75, 032304.

[7] C. H. Bennett and G. Brassard (1984) Quantum cryptography: Public key distribution and coin tossing. In Proceedings of IEEE International Conference on Computers, Systems, and Signal Processing, pages 175179, Bangalore, India, 1984. IEEE, New York.

[8] H. R. Brown and G. Svetlichny (1990) Nonlocality and Gleason's lemma. Part I. Deterministic theoriesFoundations of Physics 20, 1379-1387.

[9] G. Chiribella, G. M. D'Ariano and P. Perinotti (2010) Informational derivation of quantum theory. Physical Review A 84, 012311. arXiv: 1011.6451

[10] B. Coecke and R. Duncan (2008) Interacting quantum observables. In: Proceedings of the 35th International Colloquium on Automata, Languages and Programming (ICALP), pp. 298-310, Lecture Notes in computer science 5126, Springer-Verlag. Extended version (2011):

[11] B. Coecke and R. Duncan (2011) Interacting quantum observables: categorical algebra and diagrammatics. New Journal of Physics 13, 043016. arXiv:0906.4725

[12] B. Coecke and B. Edwards (2011) Toy quantum categories. In: Proceedings of Quantum Physics and Logic/Development of Computational Models (QPL-DCM). Electronic Notes in Theoretical computer science 271 (1), 29-40. arXiv:0808.1037

[13] B. Coecke, B. Edwards and R. W. Spekkens (2011) Phase groups and the origin of non-locality for qubits. Electronic Notes in Theoretical Computer Science 271 (2), 15-36. arXiv:1003.5005

[14] B. Coecke and A. Kissinger (2010) The compositional structure of multipartite quantum entanglement. In: Proceedings of the 37th International Colloquium on Automata, Languages and Programming (ICALP), pp. 297-308, Lecture Notes in computer science 6199, Springer-Verlag. arXiv: 1002.2540

[15] B. Coecke and E.O. Paquette (2011) Categories for the Practicing Physicist. In: New Structures for Physics, B. Coecke (Ed.), pages 173286. Springer Lecture Notes in Physics vol. 813.

[16] B. Coecke, E. O. Paquette and D. Pavlovic (2009) Classical and quantum structuralism. In: Semantic Techniques for Quantum Computation, I. Mackie and S. Gay (eds), pages 29-69, Cambridge University Press. arXiv:0904.1997

[17] B. Coecke and D. Pavlovic (2007) Quantum measurements without sums. In: Mathematics of Quantum Computing and Technology, G. Chen, L. Kauffman and S. Lamonaco (eds), pages 567-604. Taylor and Francis. arXiv:quant-ph/0608035

[18] B. Coecke, D. Pavlovic and J. Vicary (2008) A new description of orthogonal bases. To appear in Mathematical Structures in computer science. arXiv:0810.0812

[19] D. G. B. J. Dieks (1982) Communication by EPR devices. Physics Letters A 92, 271-272.

[20] R. Duncan and S. Perdrix (2010) Rewriting measurement-based quantum computations with generalised flow. In: Proceedings of the 37th International Colloquium on Automata, Languages and Programming (ICALP), Lecture Notes in computer science 6199, Springer-Verlag.

[21] B. Edwards (2009) Non-locality in Categorical Quantum Mechanics. DPhil thesis, University of Oxford. 
[22] B. Edwards (2010) Phase groups and local hidden variables. Research Report RR-10-15, Dept. of computer science, University of Oxford.

[23] A. Ekert (1991) Quantum cryptography based on Bell's theorem. Physical Review Letters 67, 661-663.

[24] D. Gottesman (1996) A class of quantum error-correcting codes saturating the quantum Hamming bound. Physical Review A 54, 1862-1868. arXiv:quant-ph/9604038

[25] D. M. Greenberger, M. A. Horne, A. Shimony and A. Zeilinger (1990) Bell's theorem without inequalities. American Journal of Physics 58, 1131-1143.

[26] L. Hardy (2011) Foliable operational structures for general probabilistic theories. In: Deep Beauty: Understanding the Quantum World through Mathematical Innovation. H. Halvorson (ed.), pages 409-442, Cambridge University Press. arXiv:0912.4740

[27] P. Heywood and M. L. G. Redhead (1983) Nonlocality and the KochenSpecker paradox. Foundations of Physics 13, 481-499.

[28] A. Hillebrand (2011) Quantum protocols involving multiparticle entanglement and their representations in the zx-calculus. MSc. thesis, University of Oxford. http://www.cs.ox.ac.uk/people/bob.coecke/Anne.pdf

[29] M. Hillery, V. Bužek, and A. Berthiaume (1999) Quantum secret sharing. Physical Review A 59, 1829-1834.

[30] C. Horsman (2011) Quantum picturalism for topological cluster-state computing. New Journal of Physics 13 095011. arXiv:1101.4722

[31] G. M. Kelly and M. L. Laplaza (1980) Coherence for compact closed categories. Journal of Pure and Applied Algebra 19, 193-213.

[32] A. Kissinger (2012) Pictures of Processes. DPhil thesis, University of Oxford.

[33] S. Lack (2004) Composing PROPs. Theory and Applications of Categories 13, 147-163.

[34] N. D. Mermin (1990) Quantum mysteries revisited. American Journal of Physics 58, 731-734.

[35] M.A. Nielsen and I.L. Chuang (2000) Quantum Computation and Quantum Information. Cambridge University Press.

[36] A. K. Pati and S. L. Braunstein (2000) Impossibility of deleting an unknown quantum state. Nature 404, 164-165. arXiv:quant-ph/9911090

[37] D. Pavlovic (2009) Ouantum and classical structures in nondeterminstic computation. Lecture Notes in computer science 5494, page 143-157, Springer. arXiv:0812.2266

[38] M. Pawlowski, T. Paterek, D. Kazlikowski, V. Scarani, A. Winter and M. Zukowski (2009) Information causality as a physical principle. Nature 461, 1101. arXiv:0905.2292

[39] E. Schrödinger (1935) Discussion of probability relations between separated systems. Proceedings of the Cambridge Philosophical Society 31, 555-563.

[40] P. Selinger (2007) Dagger compact closed categories and completely positive maps. Electronic Notes in Theoretical computer science 170, pages 139-163.

[41] P. Selinger (2011) A survey of graphical languages for monoidal categories. In: New Structures for Physics, B. Coecke (ed.), pages 289356. Lecture Notes in Physics 813, Springer-Verlag. arXiv:0908.3347

[42] R. W. Spekkens (2007) Evidence for the epistemic view of quantum states: A toy theory. Physical Review A 75, 032110. arXiv:quant-ph/0401052

[43] R. Street (2007) Quantum Groups: A Path to Current Algebra. Cambridge University Press.

[44] W. K. Wootters and W. Zurek (1982) A single quantum cannot be cloned. Nature 299, 802-803. 